\title{
ALGEBRAIC STRING OPERATIONS
}

\author{
THOMAS TRADLER AND MAHMOUD ZEINALIAN
}

\begin{abstract}
We first discuss how open/closed chord diagrams, both with and without marked points, act on appropriate Hochschild complexes possibly coupled with the two-sided cobar complex. Then, in the main part of the paper, we introduce the notion of a $\mathrm{V}_{k^{-}}$ algebra and obtain suitable homotopy versions.
\end{abstract}

\section{Contents}

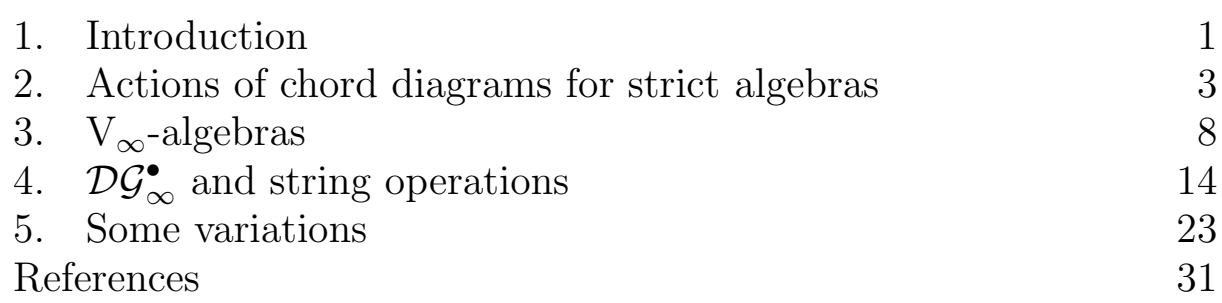

\section{INTRODUCTION}

It is long known that the Hochschild cohomology of an associative algebra has the structure of a Gerstenhaber algebra; see $G$. In recent years, it has become clear that this is only a starting point for the study of the algebraic structure of the Hochschild complex. Deligne's conjecture states that the operad of chains on the little disc operad acts on the Hochschild cochain complex of an associative algebra. In the more general case of $\mathrm{A}_{\infty}$-algebras, this conjecture was proved in KoSo. The original associative case was also proved in Kau, MS1, [Ta1, [Ta2, V]. The discovery of String Topology [CS1] suggested that in the presence of an appropriate notion of Poincaré duality a richer collection of operations should exist. In fact, under suitable assumptions, there is a plethora of operations both in the Hochschild and cyclic Hochschild settings.

A step in this direction was taken in our previous paper [TZ2], where the Hochschild complex of an associative algebra with an invariant and symmetric co-inner product was treated. We will recall the action of the cyclic Sullivan chord diagrams on the Hochschild complex of an 


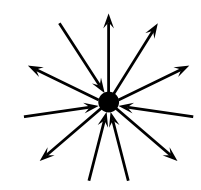

FiguRE 1. The general vertex

associative algebra with values in its dual from [TZ2] and extend the discussion to the action of the chord diagrams without marked points on the cyclic Hochschild complex, as well as the action of open/closed chord diagrams on the cyclic Hochschild complex coupled with the twosided cobar complex. The main part of this paper is a study of the algebraic structure of the cyclic Hochschild complex of an $\mathrm{A}_{\infty}$-algebra with an appropriate homotopy co-inner product. An appropriate homotopy version of an invariant and symmetric co-inner product is formulated using the notion of a $\mathrm{V}_{\infty}$-algebra. This consists of a system of elements labelled by vertices with cyclically ordered directed edges, as shown in Figure 11. For each such vertex, with $m$ incoming edges shuffled in between $n$ outgoing edges according to a partition $m=i_{1}+\cdots+i_{n}$, we associate an element of $A \otimes\left(A^{*}\right)^{\otimes i_{1}} \otimes \cdots \otimes A \otimes\left(A^{*}\right)^{\otimes i_{n}}$, where $A$ is the underlying vector space of our algebra, and $A^{*}$ its dual. These elements must satisfy certain conditions, which are stated in Definition 3.1. If we restrict the structure to vertices with only one outgoing edge, then we recover the notion of an $\mathrm{A}_{\infty}$-algebra. In general, for a fixed $k=1,2, \cdots, \infty$, we consider $\mathrm{V}_{k}$-algebras, governed by of vertices with $m$ incoming and $n$ outgoing edges, where $m$ is arbitrary while $n<k+1$. In general, there are obstructions to completing a $\mathrm{V}_{k^{-}}$ algebra to a $V_{k+1}$-algebra. Interesting examples of $\mathrm{V}_{k}$-algebras occur in topology. For instance, $V_{2}$-algebras, which are $\mathrm{A}_{\infty}$-algebras with an invariant and symmetric homotopy co-inner product, can be constructed from a triangulated and oriented Poincaré duality space; see TZ1.

We will describe graphs which generalize cyclic Sullivan Chord diagrams and construct a graph complex, denoted by $\mathcal{D} \mathcal{G}_{k}^{\bullet}$. Roughly speaking, $\mathcal{D} \mathcal{G}_{k}^{\bullet}$ is generated by the set of directed graphs with vertices having at most $k$ outgoing edges and has the structure of a PROP. Equipped with these notions, we show how the cyclic Hochschild complex of a $\mathrm{V}_{k}$-algebra has a natural action of $\mathcal{D} \mathcal{G}_{k}^{\bullet}$, for $k=1,2, \cdots, \infty$. The case of $k=1$ is somewhat akin to what was considered in KoSo] for the Hochschild complex of an $\mathrm{A}_{\infty}$-algebra. In this paper, we treat the cyclic Hochschild complex in detail because it is very clean and 
the graphs do not carry any extra decorations such as marked points. We will then discuss modifications needed to address the open/closed string interactions, i.e., operation on the cyclic Hochschild complex coupled with the two-sided cobar complex, as well as the case in which the cyclic Hochschild complex is replaced by the Hochschild complex.

Here is a short description of the individual sections. Section 2 is devoted to strictly associative algebras with an invariant and symmetric co-inner products. The open/closed case and the cases of chord diagrams both with and without marked points will be investigated. In section 3, the notion of $\mathrm{V}_{k}$-algebras will be defined and several special cases will be examined. In section 4 , we define the PROP $\mathcal{D} \mathcal{G}_{k}^{\bullet}$ and show that it acts on the cyclic Hochschild complex of a $V_{k}$-algebra. Sections 3 and 4 form the core of this paper. In section 5 , we discuss the modifications necessary to obtain the open/closed and the nonequivariant operations for homotopy algebras. Also, the relevance of the algebraic structures considered in this paper to topology will be explored.

Acknoledgements. We would like to thank Dennis Sullivan and Jim Stasheff for their useful comments.

\section{ACtions OF CHORD DIAGRAMS FOR STRICT ALGEBRAS}

This paper mainly treats chain level operations on the cyclic Hochschild complex and the Hochschild complex of $\mathrm{V}_{\infty}$-algebras. It is useful, though, to start by describing the operations in the simple case of an ungraded and strictly associative algebras. We will first recall from [TZ2, how chord diagrams with marked points act on the Hochschild complex. We then describe a cyclic version in which chord diagrams without marked points act on the cyclic Hochschild complex of a strictly associative algebra. We finish the section by incorporating the twosided cobar complex into the picture.

2.1. Action of chord diagrams on Hochschild complex. Recall from [TZ2 that a cyclic Sullivan chord diagram consists of circles and chords, where the endpoints of the chords lie on the circles. A thickening of the chord diagram will give rise to a surface with two types of boundaries. Those situated inside of the circles are referred to as the inputs while the remaining ones form the outputs. Both inputs and outputs are numerated, and there is exactly one marked point for each input and output. It may also happen that an input marked point and an output marked point are attached at the same spot, or that a marked point may be attached to an endpoint of a chord. For further 


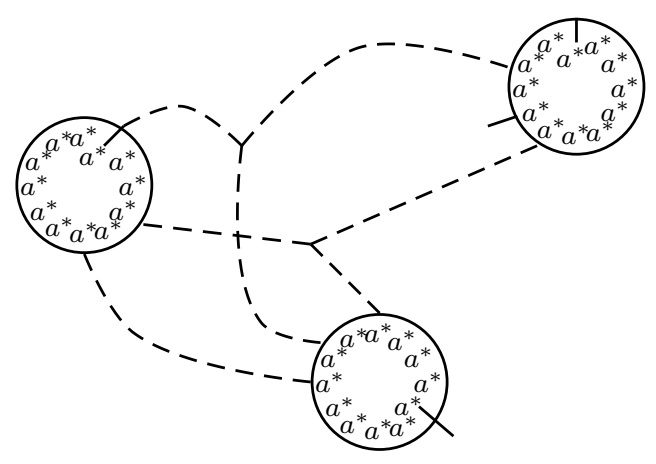

Figure 2. Chord diagrams with marked points

details on how to treat these cases see [TZ2. An example of such a chord diagram is given in Figure 2 .

Now, let $A$ be an associative algebra with unit $\mathbf{1}$ and an invariant and symmetric co-inner product $U=\sum a_{i} \otimes b_{i} \in A \otimes A$. By definition, symmetric means $\sum a_{i} \otimes b_{i}=\sum b_{i} \otimes a_{i}$ and invariant means $\sum a_{i} a \otimes b_{i}=$ $\sum a_{i} \otimes a b_{i}$ and $\sum a a_{i} \otimes b_{i}=\sum a_{i} \otimes b_{i} a$, for all $a \in A$. Note that an invariant and symmetric inner product that is nondegenerate gives rise to a co-inner product. The Hochschild cochain complex of $A$ with values in its dual $A^{*}$ is by definition the graded vector space,

$$
C H^{\bullet}\left(A, A^{*}\right)=\prod_{j=0}^{\infty}\left\{f: A^{\otimes j} \rightarrow A^{*}\right\},
$$

with the differential,

$$
\begin{aligned}
\delta f\left(a_{1}, \cdots, a_{j}\right)= & a_{1} \cdot f\left(a_{2}, \cdots, a_{j}\right) \\
& +\sum_{i=1}^{j-2}(-1)^{i} f\left(a_{1}, \cdots, a_{i} a_{i+1}, \cdots, a_{j}\right) \\
& +(-1)^{j-1} f\left(a_{1}, \cdots, a_{j-1}\right) \cdot a_{j} .
\end{aligned}
$$

In the presence of a unit, one may furthermore define the normalized subcomplex,

$$
\begin{aligned}
\overline{C H^{\bullet}}\left(A, A^{*}\right) & =\prod_{j=0}^{\infty}\left\{f: A^{\otimes j} \rightarrow A^{*} \mid\right. \\
& \left.f\left(a_{1}, \cdots, a_{j}\right)=0, \text { if any of the } a_{1}, \cdots, a_{j} \text { equals } \mathbf{1}\right\} .
\end{aligned}
$$

The inclusion $\overline{C H^{\bullet}}\left(A, A^{*}\right) \hookrightarrow C H^{\bullet}\left(A, A^{*}\right)$ is a quasi-isomorphism; see L. 


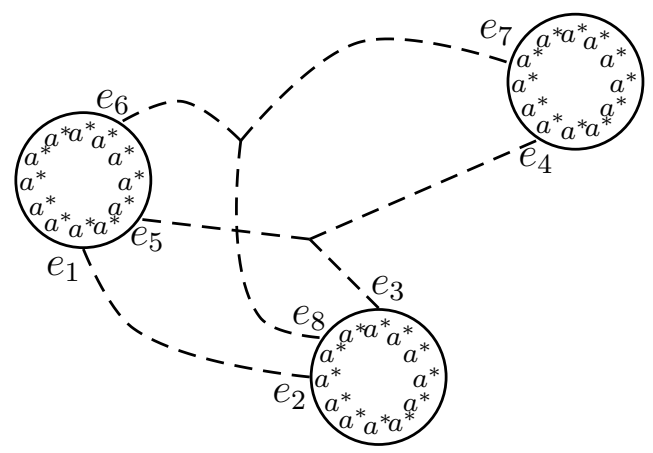

Figure 3. Chord diagrams without marked points

It was shown in [TZ2] that the PROP of cyclic Sullivan chord diagrams with marked points acts on the normalized Hochschild cochain complex by applying elements of $\overline{C H^{\bullet}}\left(A, A^{*}\right)$ to the inputs of a chord diagram, where the special last element is lined up with the input marked point. The outputs of the chord diagram yield the output of the operation, where the element aligned with the output marked point is interpreted as the special last element. The role of a chord is to apply the co-inner product to the elements at its endpoints.

\subsection{Action of chord diagrams on cyclic Hochschild complex.}

Next, we will describe a cyclic version of the above action. For this, we will look at a simpler class of chord diagrams, namely chord diagrams similar to the ones in Figure 2, with the distinction of not having marked points. Thus, the circles and chords determine the inputs and outputs, but there are no longer marked points to determine the position of a special element. An example of such a chord diagram is shown in Figure 3. In addition to the combinatorics given by the chord diagram, we require that each diagram comes with an orientation on the vector space generated by the chords endpoints. For example, the chord diagram in Figure 3 has eight chord endpoints, labelled by $e_{1}, \cdots, e_{8}$, and a possible orientation could be given by $e_{1} \wedge \cdots \wedge e_{8}$. A chord diagram with an orientation is identified with the negative of the same diagram with the opposite orientation.

The boundary of a chord diagram is given by a sum obtained by collapsing each of the circle pieces in between the endpoints of the chords, one at a time. The induced orientation for each collapse is determined by bringing the two chord endpoints of the collapsing circle to the beginning of the expression for the orientation, in the order determined by the clockwise direction of the circle piece, and then 
fusing them into one point. For example, if we would like to collapse the the circle piece between $e_{2}$ and $e_{8}$ in Figure 3. then we write $e_{1} \wedge$ $\cdots \wedge e_{8}=-e_{2} \wedge e_{8} \wedge e_{1} \wedge e_{3} \wedge \cdots \wedge e_{7}$ and take the induced orientation $-e_{9} \wedge e_{1} \wedge e_{3} \wedge \cdots \wedge e_{7}$, where $e_{9}$ is the chord endpoint obtained by fusing $e_{2}$ and $e_{8}$.

We may also compose two chord diagrams. This consists of identifying the outputs of the first diagram with the inputs of the second diagram, and then attaching the chords of the second diagram to the first diagram in all possible ways. If $e_{1} \wedge \cdots \wedge e_{n}$ is the orientation of the first diagram and $e_{n+1} \wedge \cdots \wedge e_{m}$ is the orientation of the second diagram, then $e_{1} \wedge \cdots \wedge e_{n} \wedge e_{n+1} \wedge \cdots \wedge e_{m}$ is the orientation of the composition of the diagrams.

Now, let $A$ be an associative algebra concentrated in degree zero, with an invariant and symmetric co-inner product $U$. That is to say, $U=\sum a_{i} \otimes b_{i} \in A \otimes A$ satisfies

(1) $\sum a_{i} \otimes b_{i}=\sum b_{i} \otimes a_{i}$

(2) $\sum a_{i} a \otimes b_{i}=\sum a_{i} \otimes a b_{i}$, for every $a \in A$,

(3) $\sum a a_{i} \otimes b_{i}=\sum a_{i} \otimes b_{i} a$, for every $a \in A$.

We consider the cyclic Hochschild cochain complex,

$$
\begin{aligned}
C C^{\bullet}(A)=\prod_{j=0}^{\infty}\left\{f: A^{\otimes j+1} \rightarrow \mathbf{k} \mid\right. \\
\left.\quad f\left(a_{1}, \cdots, a_{j+1}\right)=(-1)^{j} \cdot f\left(a_{j+1}, a_{1}, \cdots, a_{j}\right)\right\},
\end{aligned}
$$

with the differential,

$$
\begin{aligned}
\delta f\left(a_{0}, \cdots, a_{j}\right)= & \sum_{i=0}^{j-1}(-1)^{i} f\left(a_{0}, \cdots, a_{i} a_{i+1}, \cdots, a_{j}\right) \\
& +(-1)^{j}\left(a_{j} a_{0}, \cdots, a_{j-1}\right) .
\end{aligned}
$$

To better understand the signs in the above expression, it would be more conceptual to think that elements of $A$ have been shifted to degree -1 , and that the signs are those coming from by the Koszul sign rule which introduces a $(-1)^{p q}$, whenever something of degree $p$ is moved passed something of degree $q$.

We claim that the PROP of chord diagrams without marked points acts on $C C^{\bullet}(A)$. In fact, this action is very similar to the action on $\overline{C H^{\bullet}}\left(A, A^{*}\right)$ from the previous section. Elements $f_{1}, \cdots, f_{k}$ of $C C^{\bullet}(A)$ will be applied to the input circles of the chord diagram. We may write the inputs $f_{1} \otimes \cdots \otimes f_{k}$ in a linear order staring from the first to the $k^{\text {th }}$ input. Then, much like before, for each chord, the co-inner 


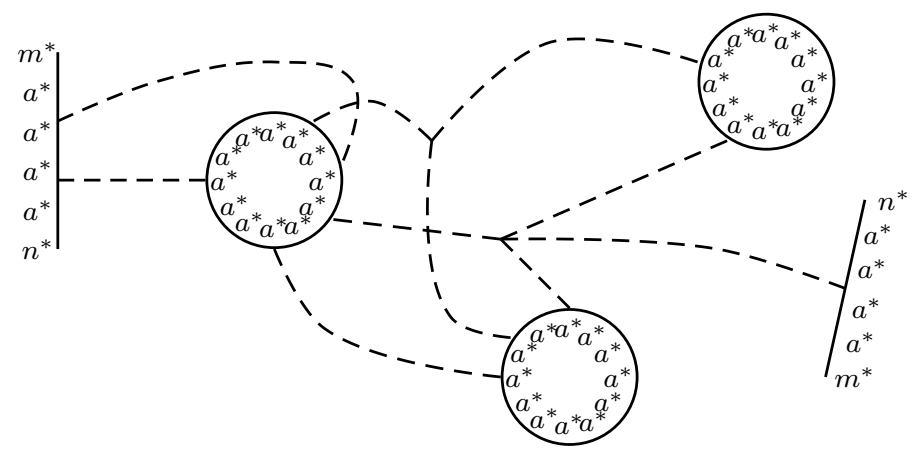

Figure 4. Open/closed chord diagrams without marked points

product is applied to the elements at the chords endpoints. To obtain the correct signs, before applying the co-inner product to the elements of the shifted $A^{*}$, one needs to shift them back to degree zero. In order to unshift them, we apply shift operators of degree 1 to each chord endpoint. More precisely, we apply shift operations on the left of $f_{1} \otimes \cdots \otimes f_{k}$ in the order given by the orientation $e_{1} \wedge \cdots \wedge e_{n}$ of the diagram. Then, the inner products for the chords may safely be applied, since the chord endpoints will now have degree zero. We collect the outputs of the chord diagram as outputs of the operation. Since these outputs will in general not be cyclically invariant, we perform a final cyclic symmetrization to each output.

\subsection{Action of open/closed chord diagrams on cyclic Hochschild} complex coupled with two-sided cobar complex. As yet another variation, we describe an action of the open/closed chord diagrams. For this, we look at chord diagrams without marked points, with some of the circles opened up to intervals. Thus, the open/closed chord diagrams consist of intervals, circles and chords; see Figure 4. Since there are no marked points, we additionally require an orientation on the vector space generated by the chords endpoints.

Once again, let $A$ be an associative algebra with an invariant and symmetric co-inner product $U \in A \otimes A$, and let $M$ and $N$ be left and right modules over $A$, respectively. Consider the two-sided cobar complex,

$$
C^{\bullet}(M, A, N)=\prod_{j=0}^{\infty}\left\{f: M \otimes A^{\otimes j} \otimes N \rightarrow \mathbf{k}\right\}
$$

Using the left and right module structures on $M$ and $N$, one defines a differential on $C^{\bullet}(M, A, N)$; see section 5.1 . 
We claim that the PROP of open/closed chord diagrams acts on $C C^{\bullet}(A) \otimes C^{\bullet}(M, A, N)$. Much like before, $C C^{\bullet}(A)$ corresponds to the closed circles, and $C^{\bullet}(M, A, N)$ to the open intervals. To be more precise, we associate $M$ to the left endpoint and $N$ to the right endpoint of the interval. Once again, chords will apply co-inner products, and we can collect the outputs according to the outputs of the diagram. An orientation on the vector space generated by the chords endpoints is needed to determine the order in which the inverse of the shift operator, bringing elements of $A^{*}$ from the shifted degree +1 back to degree 0 , must be applied.

\section{3. $\mathrm{V}_{\infty}$-ALGEBRAS}

One would like to extend the above discussions to a category in which associative algebras are replaced by $\mathrm{A}_{\infty}$-algebras. Although the notion of homotopy co-inner products proves helpful in the study of Hochschild cohomology $H H^{\bullet}\left(A, A^{*}\right)$ of an $\mathrm{A}_{\infty}$-algebra $A$ (see $\operatorname{Tr} 2$ ) there are limitations when it comes to Hochschild cochains $C H^{\bullet}\left(A, A^{*}\right)$. These limitations seem to be more pronounced in the cyclic case of $C C^{\bullet}(A)$, as we will point out in section 4.5. In fact, in order to see that the cyclic Hochschild cohomology is a Lie algebra, higher homotopies that are not part of the homotopy co-inner product are needed to guarantee the Jacobi identity.

In this section, we describe the concept of a $\mathrm{V}_{k}$-algebra, which is germane to the study of the algebraic structure of the Hochschild complex and cyclic Hochschild complex of an $\mathrm{A}_{\infty}$-algebra. The notion of an $\mathrm{A}_{\infty}$-algebra is only the first layer of this structure. The second layer is where the concept of an invariant and symmetric homotopy coinner product comes into its own. The third and higher layers are new concepts that we shall develop and use. We will see in section 4 how considering $\mathrm{V}_{k}$-algebras will shed some light on the algebraic structure of the cyclic Hochschild complex and its cousins.

3.1. $\mathbf{A}_{\infty}$-algebras or $V_{1}$-algebras. Let $\left(A=\bigoplus_{n \in \mathbb{Z}} A_{n}, \partial_{A}\right)$ be a complex over a field $\mathbf{k}$ of characteristic zero, such that each $A_{n}$ is a finite dimensional vector space. Consider the dual complex $\left(A^{*}=\bigoplus_{n \in \mathbb{Z}} A_{n}^{*}, \partial_{A}^{*}\right)$. Note that the grading for the dual space $A^{*}$ is negative of that for $A$. For instance, if $A$ is concentrated in negative degrees, then $A^{*}$ is positively graded. The finite dimensionality assumption allows for such identifications as $\operatorname{Hom}(A, A)=A^{*} \otimes A$ as graded objects. An associative algebra structure on $A$ consists of a chain map $\mu_{2}: A^{\otimes 2} \rightarrow A$, usually written as $\mu_{2}\left(a_{1}, a_{2}\right)=a_{1} \cdot a_{2}$, such that $\left(a_{1} \cdot a_{2}\right) \cdot a_{3}=a_{1} \cdot\left(a_{2} \cdot a_{3}\right)$. A representation of the identities for associativity using graphs is shown 


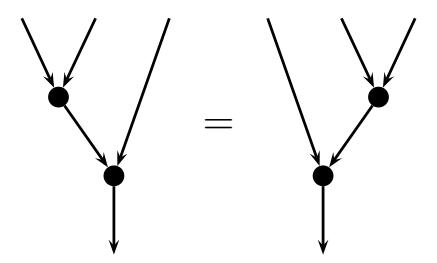

Figure 5. Associativity

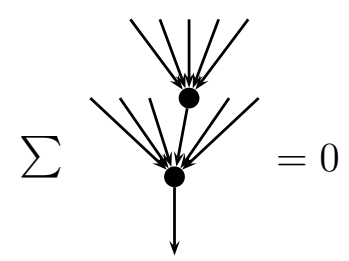

Figure 6. Homotopy associativity $\left(\mathrm{A}_{\infty}\right)$

in Figure 5, where incoming edges denote the arguments of $\mu_{2}$ and an outgoing edge stands for its output.

The concept of an $\mathrm{A}_{\infty}$-algebra, introduced by J. Stasheff in $\mathrm{St}$, is a generalization of the concept of associative algebra. An $\mathrm{A}_{\infty}$-algebra is a vector space $A$ endowed with a sequence of maps $\left\{\mu_{n}: A^{\otimes n} \rightarrow A\right\}_{n \geq 1}$, where the sum of all compositions of any $\mu_{i}$ and $\mu_{j}$ is zero,

$$
\sum_{i, j, k} \pm \mu_{i}\left(a_{1}, \cdots, \mu_{j}\left(a_{k}, \cdots, a_{k+j-1}\right), \cdots, a_{n}\right)=0 .
$$

We defer the discussion of signs until section 3.3. where we cover the general case. A graphical representation of the above identities can be seen in Figure 6. Here, the $j$ incoming edges stand for the arguments of $\mu_{j}$ and the one outgoing edge is used for its output. Put slightly differently, we may view $\mu_{j}: A^{\otimes j} \rightarrow A$ as an element $\mu_{j} \in A \otimes\left(A^{*}\right)^{\otimes j}$, and reserve the incoming edges for the elements of $A^{*}$, and the outgoing edge for those of $A$. This assignment of elements to the edges, which is suitable for evaluating of elements of $A^{*}$ on $A$, is used throughout the paper.

3.2. $\mathbf{A}_{\infty}$-algebras with homotopy co-inner products or $V_{2}$-algebras. We also need a generalization of the concept of an invariant and symmetric co-inner product. By definition, an invariant and symmetric co-inner product of dimension $d$ on a graded associative algebra $A$ (finite dimensional in each degree, and without a unit) is an element 


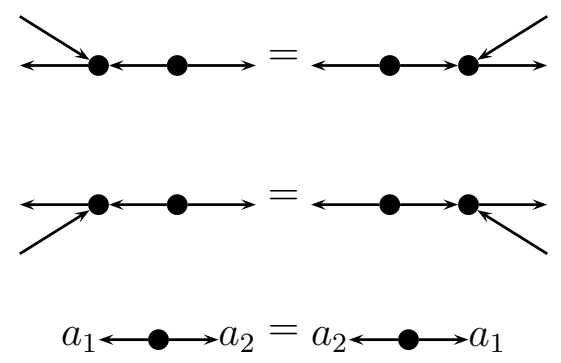

Figure 7. Co-inner product

$U=\sum a_{i} \otimes b_{i} \in A \otimes A$ of degree $-d$, called the co-inner product, satisfying,

(1) $\sum a_{i} \otimes b_{i}=\sum \pm b_{i} \otimes a_{i}$

(2) $\sum a_{i} a \otimes b_{i}=\sum \pm a_{i} \otimes a b_{i}$, for every $a \in A$,

(3) $\sum a a_{i} \otimes b_{i}=\sum \pm a_{i} \otimes b_{i} a$, for every $a \in A$,

where the signs \pm are those dictated by the Koszul sign rule.

In practice, co-inner products of dimension $d$ are often obtained from inner products $\langle\cdot, \cdot\rangle: A \otimes A \rightarrow \mathbf{k}$, of degree $d$, such that,

$\left(1^{\prime}\right)\langle a, b\rangle=\langle a, b\rangle$

$\left(2^{\prime}\right)\langle a b, c\rangle=\langle a, b c\rangle$,

$\left(3^{\prime}\right)\langle a, b c\rangle=\langle c a, b\rangle$,

$\left(4^{\prime}\right) A \rightarrow A^{*}$, defined by $a \mapsto\langle a, \cdot\rangle$, is an isomorphism, for every $a \in A$.

Thus, sing the isomorphism $A \cong A^{*}$ one transports the inner product $\langle\cdot, \cdot\rangle \in A^{*} \otimes A^{*}$ to get an element of $U \in A \otimes A$.

For instance, for a Poincaré duality space $X$ of dimension $d$, consider the cohomology $H^{\bullet} X$, negatively graded, and define $\langle\cdot, \cdot\rangle: H^{\bullet} X \times$ $H^{\bullet} X \rightarrow \mathbf{k}$ by $\langle a, b\rangle=(a \cup b)[X]$. The co-inner product is given by the Thom class of the diagonal $U \in H^{\bullet} X \otimes H^{\bullet} X$.

In terms of graphs, a co-inner product $U \in A \otimes A$ is represented by a vertex with two outgoing edges. Figure 7 represents the required identities in terms of graphs. Notice that the cyclic ordering of the arguments $a_{1}^{*}, a_{2}^{*}$ and $a$ are preserved in each of the equations.

Just as associative algebras were generalized to $\mathrm{A}_{\infty}$-algebras, coinner products may be generalized to homotopy co-inner products. A treatment of such a generalization for any cyclic operad appears in [LT. As it turns out, the homotopy co-inner products are given by elements $v_{i, j} \in A \otimes\left(A^{*}\right)^{\otimes i} \otimes A \otimes\left(A^{*}\right)^{\otimes j}$. These $v_{i, j}$ 's satisfy generalized co-inner product conditions indicated in Figure 8 . In Figure 8, the upper equation is the generalized bimodule condition. Here, each $v_{i, j}$ 


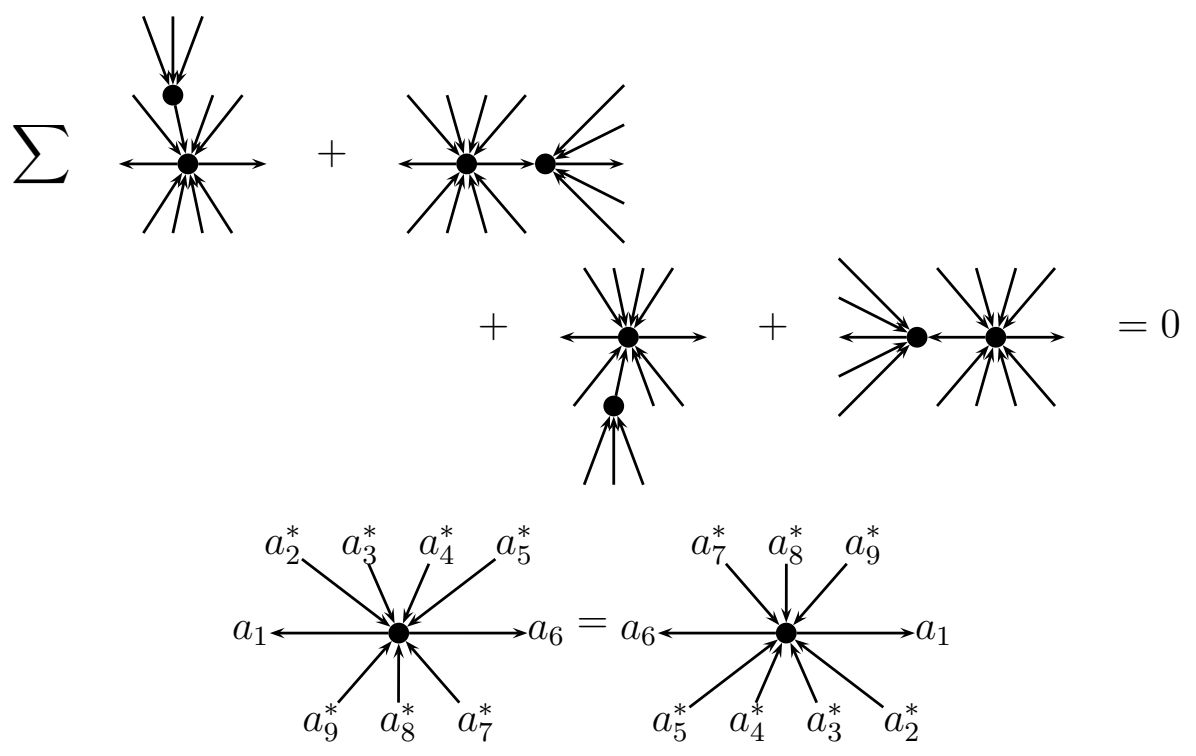

FiguRE 8. Homotopy co-inner product

is pictured by a vertex with two (horizontal) outgoing edges, $i$ incoming edges on top, and $j$ incoming edges at the bottom. For each $i, j \geq 0$, we go around $v_{i, j}$ and for all $m \geq 1$ attach the maps $\mu_{m}$ of the $\mathrm{A}_{\infty}$-algebra structure in all possible ways. Notice the difference between attaching a $\mu_{m}$ to one of the horizontal arrows as opposed to attaching it to one from the top or the bottom. It is also worth mentioning that the above sum splits into countably many equations, each of which is a finite sum with a fixed number of edges. The lower equation in Figure 8 shows how the symmetry condition is generalized. It is simply by requiring that each $v_{i, j}$ is related to $v_{j, i}$ via a $180^{\circ}$ rotation.

3.3. $\mathbf{V}_{k}$-algebras, for $\mathbf{1} \leq \mathbf{k} \leq \infty$. In previous sections, we looked at algebraic objects that were labelled graphically by vertices with an arbitrary number of incoming edges but only one or at most two outgoing ones. We call these vertices of types 1 and 2, respectively. More generally, a vertex of type $n$ has an unrestricted number of incoming edges shuffled in between $n$ outgoing edges. The incoming edges correspond to elements of $A^{*}$, whereas the ones outgoing are labelled with elements of $A$. 

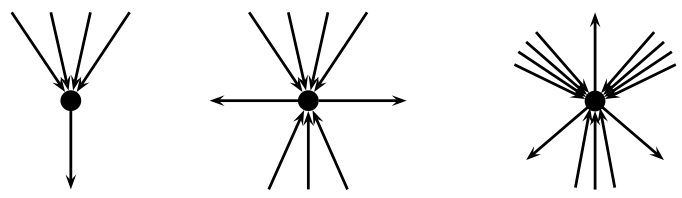

Figure 9. Vertices of type 1, 2 and 3

$$
\begin{array}{lll}
\text { type } 1 \leftrightarrow & \mu_{j} \in A \otimes\left(A^{*}\right)^{\otimes j} & \leftrightarrow A_{\infty} \text {-algebra } \\
\text { type } 2 \leftrightarrow & v_{i, j} \in A \otimes\left(A^{*}\right)^{\otimes i} \otimes A \otimes\left(A^{*}\right)^{\otimes j} & \leftrightarrow \text { Homotopy } \\
& & \text { co-inner product }
\end{array}
$$

We now consider algebraic objects that correspond to vertices of type $n$, for $n \in \mathbb{N}$. Each vertex of type $n$ labels an element $v_{i_{1}, \cdots, i_{n}} \in$ $A \otimes\left(A^{*}\right)^{\otimes i_{1}} \otimes \cdots \otimes A \otimes\left(A^{*}\right)^{\otimes i_{n}}$ for $i_{1}, \cdots, i_{n} \in \mathbb{N} \cup\{0\}$, satisfying conditions analogous to those in Figure 8

Definition 3.1. For fixed $k \in\{1,2,3, \cdots, \infty\}$, a $\mathrm{V}_{k}$-algebra structure of dimension $d$ on $A$ consists of a sequence of elements,

$$
\begin{aligned}
v_{i_{1}} & \in A \otimes\left(A^{*}\right)^{\otimes i_{1}} \quad, i_{1} \neq 0 \\
v_{i_{1}, i_{2}} & \in A \otimes\left(A^{*}\right)^{\otimes i_{1}} \otimes A \otimes\left(A^{*}\right)^{\otimes i_{2}} \\
& \vdots \\
v_{i_{1}, \cdots, i_{k}} & \in A \otimes\left(A^{*}\right)^{\otimes i_{1}} \otimes \cdots \otimes A \otimes\left(A^{*}\right)^{\otimes i_{k}}
\end{aligned}
$$

for any indices $i_{1}, \cdots, i_{n} \in \mathbb{N} \cup\{0\}$, where $1 \leq n<k+1$, subject to the following conditions.

- Degree: Each $v_{i_{1}, \cdots, i_{k}}$ is an element of degree $n(2-d)+(d-4)$, with respect to the grading of $A \otimes\left(A^{*}\right)^{\otimes i_{1}} \otimes \cdots \otimes A \otimes\left(A^{*}\right)^{\otimes i_{k}}$ obtained by adding -1 to the degrees of the elements of $A^{*}$, but leaving the degree of elements of $A$ unshifted.

- Symmetry condition: If $\tau: A \otimes\left(A^{*}\right)^{\otimes i_{1}} \otimes A \otimes\left(A^{*}\right)^{\otimes i_{2}} \otimes A \otimes \cdots \otimes$ $A \otimes\left(A^{*}\right)^{\otimes i_{n}} \rightarrow A \otimes\left(A^{*}\right)^{\otimes i_{n}} \otimes A \otimes\left(A^{*}\right)^{\otimes i_{1}} \otimes \cdots \otimes A \otimes\left(A^{*}\right)^{\otimes i_{n-1}}$ denotes the cyclic rotation of tensor factors, then,

$$
v_{i_{2}, \cdots, i_{n}, i_{1}}=(-1)^{\epsilon} \cdot \tau\left(v_{i_{1}, i_{2}, \cdots, i_{n}}\right)
$$

Here, $(-1)^{\epsilon}$ is obtained by the Koszul sign rule in the above grading; see Figure 10.

- Boundary condition: For fixed $1 \leq n<k+1$, and $i_{1}, \cdots, i_{n} \in$ $\mathbb{N} \cup\{0\}$, let $\Gamma=\Gamma\left(i_{1}, \cdots, i_{n}\right)$ be the set of rooted and directed trees, that are obtained by expanding exactly one edge in the unique tree given by the type $n$ vertex with $m=i_{1}+\cdots+i_{n}$ 


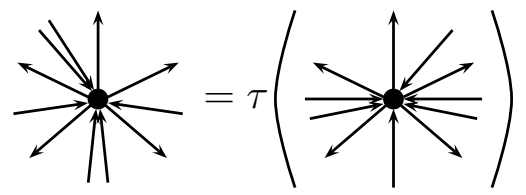

FIgURE 10. The symmetry condition

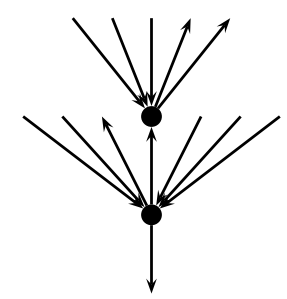

Figure 11 . A tree in $\Gamma=\Gamma(2,3,0,3)$, with $n=4$ outgoing edges

incoming edges; see Figure 11] For a tree $\gamma \in \Gamma$, there is no restriction on the orientation of the internal edge (compare for example the first two terms in Figure 12). Also, $\gamma$ has exactly two internal vertices, with $j_{1}, \cdots, j_{r}$ and $j_{1}^{\prime}, \cdots, j_{s}^{\prime}$ incoming edges, respectively. We can assign $v_{j_{1}, \cdots, j_{r}}$ and $v_{j_{1}^{\prime}, \cdots, j_{s}^{\prime}}$ to these vertices, and write them in the order given by the internal edge of $\gamma$, say $v_{j_{1}, \cdots, j_{r}} \otimes v_{j_{1}^{\prime}, \cdots, j_{s}^{\prime}}$. Then, we fuse one tensor factor of $v_{j_{1}, \cdots, j_{r}}$ with one tensor factor of $v_{j_{1}^{\prime}, \cdots, j_{s}^{\prime}}$, as indicated by the internal edge of $\gamma$. The result is denoted by $(-1)^{\epsilon} \cdot v(\gamma) \in$ $A \otimes\left(A^{*}\right)^{\otimes i_{1}} \otimes \cdots \otimes A \otimes\left(A^{*}\right)^{\otimes i_{n}}$, where $\epsilon$ is obtained by the product of shifted degrees that have switched positions in the linear expansion of $v_{j_{1}, \cdots, j_{r}} \otimes v_{j_{1}^{\prime}, \cdots, j_{s}^{\prime}}$. In this notation, we require the following boundary condition; see Figure 12.

$$
\sum_{\gamma \in \Gamma}(-1)^{\epsilon} \cdot v(\gamma)=0
$$

Remark 3.2. A few comments are in order.

(1) It is important to recall that the grading of $A^{*}$ is negative of that of $A$.

(2) The boundary condition interlocks operations of different types.

(3) In the above definition, we did not include $v_{0} \in A$ only to avid further consideration arising from the existence of a homotopy 


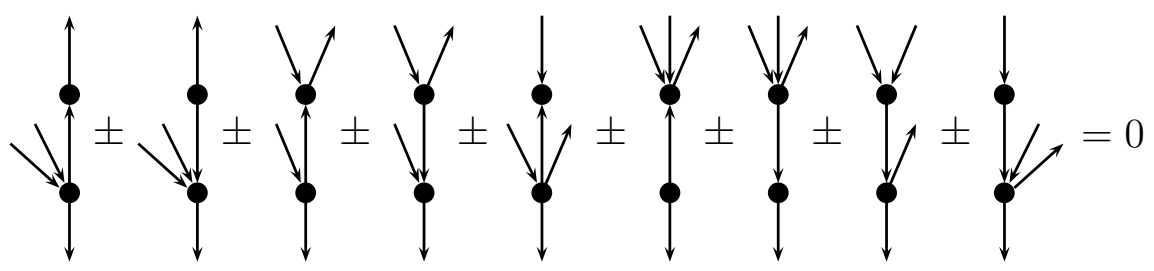

Figure 12. The boundary condition for $\Gamma=\Gamma(2,0)$

unit for weak $\mathrm{A}_{\infty}$-algebras. Nonetheless, one could consider a discussion which allows for a $v_{0} \in A$.

The concept of a $\mathrm{V}_{k}$-algebra is homotopy invariant. That is, the $\mathrm{V}_{k}$-algebra structure on a complex can be transported onto a quasiisomorphic complex. $\mathrm{A} \mathrm{V}_{1}$-algebras is precisely an $\mathrm{A}_{\infty}$-algebra, and a $\mathrm{V}_{2}$-algebra is exactly an $\mathrm{A}_{\infty}$-algebra with an invariant and symmetric homotopy co-inner product. $\mathrm{V}_{3}$-algebras are less familiar as they deal with type 3 vertices. An example of a vertex of higher type appeared in H. Kajiura's thesis Kaj, section 4.2] in connection with open strings.

\section{4. $\mathcal{D} \mathcal{G}_{\infty}^{\bullet}$ AND STRING OPERATIONS}

We now define a complex generated by graphs with vertices of arbitrary types; see section 3.3. We will show how this complex acts on the Connes' cyclic Hochschild complex. Intuitively, this generalizes the complex of cyclic Sullivan chord diagrams by adding magnifying glass information to the chords; see Figure 22.

4.1. Cyclic Hochschild complex. Let us recall the definition of the cyclic Hochschild complex. Let $A$ be a graded vector space and define the cyclic Hochschild complex $C C^{\bullet}(A)$ by,

$$
\begin{aligned}
C C^{\bullet}(A)=\prod_{j=0}^{\infty}\left\{f: A^{\otimes j+1} \rightarrow \mathbf{k} \mid\right. \\
\left.\quad f\left(a_{1}, \cdots, a_{j+1}\right)=(-1)^{\epsilon} \cdot f\left(a_{j+1}, a_{1}, \cdots, a_{j}\right)\right\},
\end{aligned}
$$

where $\epsilon=\left|a_{j+1}\right| \cdot\left(\left|a_{1}\right|+\cdots+\left|a_{j}\right|\right)$. Here, $|\cdot|$ denotes the shifted degree of an element defined as $|a|=\operatorname{deg}(a)+1$. A type zero vertex with six edges is shown in Figure 13. We will later see how an element $f \in\left(A^{*}\right)^{\otimes(j+1)} \subset C C^{\bullet}(A)$ with $j+1 \geq 6$ incoming edges can be placed on top of this vertex which plays the role of an input. If the number of tensor factors, $j+1$, exceeds 6 , then six of them are placed exactly on the top of the edges and the remanning ones are left as what will 


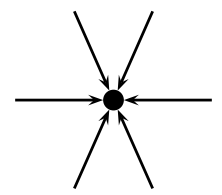

Figure 13. Cyclic Hochschild complex $C C^{\bullet}(A)$

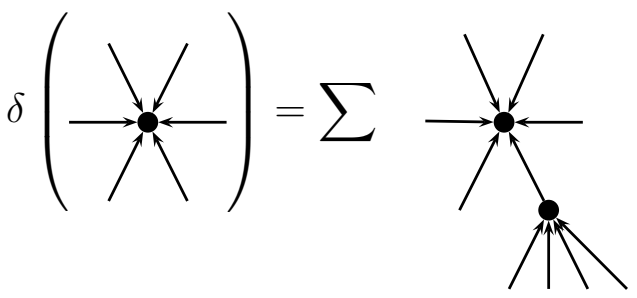

Figure 14. Differential on $C C^{\bullet}(A)$

be referred to as hairs sticking out of the vertex. This is done in all possible combinatorial ways.

As it turns out, one does not need to specify a starting edge. That is, if $f$ is a cyclic element, $f=\sum_{i=1}^{j+1} \pm a_{i}^{*} \otimes \cdots \otimes a_{j+1}^{*} \otimes a_{1}^{*} \otimes \cdots \otimes$ $a_{i-1}^{*} \in\left(A^{*}\right)^{\otimes j+1}$, then we can choose any of the $j+1$ incoming edges of Figure 13 as the first edge, and insert the $a_{1}^{*}, \cdots, a_{j+1}^{*}$ cyclically. This procedure, as we shall see, is independent of the choice of this first edge, due to cyclicity of the expressions and an overall sign obtained from an orientation.

Now, for an $\mathrm{A}_{\infty}$-algebra $A$, one can define a differential $\delta: C C^{\bullet}(A) \rightarrow$ $C C^{\bullet}(A)$ as pictured in Figure 14, To be more precise, the differential $\delta$ applies the $\mathrm{A}_{\infty}$-algebra maps $\mu_{m}$ (for $m \geq 1$ ) in all possible ways to an element $f \in C C^{\bullet}(A)$, in the following fashion,

$$
\begin{aligned}
\delta f\left(a_{1}, \cdots, a_{j+1}\right)= & \sum_{l, i} \pm f\left(\mu_{l}\left(a_{i}, \cdots, a_{j+1}, a_{1}, \cdots\right), \cdots, a_{i-1}\right) \\
& +\sum_{l, i} \pm f\left(a_{1}, \cdots, \mu_{l}\left(a_{i}, \cdots, a_{i+l-1}\right), \cdots, a_{j+1}\right) \\
& +\sum_{l, i} \pm f\left(a_{i}, \cdots, \mu_{l}\left(\cdots, a_{j+1}, a_{1}, \cdots, a_{i-1}\right)\right) .
\end{aligned}
$$

The signs are determined by comparing the linear order of the symbols in an expression to the orientation $\mu_{l} \wedge f \wedge a_{1} \wedge \cdots \wedge a_{j+1}$, where $\mu_{l}$ is of degree 1 , and $a_{i}$ has the shifted degree $\left|a_{i}\right|=\operatorname{deg}\left(a_{i}\right)+1$. Note that if $f \in\left(A^{*}\right)^{\otimes j+1}$ is cyclically invariant, then $\delta(f)$ is also cyclically 


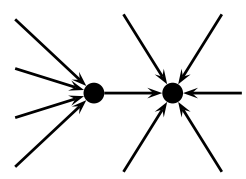

Figure 15. Plugging type 1 to type 0

invariant. Furthermore, the picture for $\delta^{2}(f)$ corresponds to applying two $\mathrm{A}_{\infty}$-algebra maps to $f$, say $\mu_{l}$ and $\mu_{m}$. Note that each term for which $\mu_{l}$ and $\mu_{m}$ sit on different edges of $f$ appears twice, obtained from first applying $\mu_{l}$ and then $\mu_{m}$, and vice versa. Since $\mu_{m}$ moves over $\mu_{l}$ in one case, but not the other, they appear with opposite signs and cancel. As for the terms where $\mu_{l}$ sits on top of $\mu_{m}$, they add up to zero due to the compatibility requirements of the $\mu_{i}$ 's in the definition of an $\mathrm{A}_{\infty}$-algebra; see section 3.1. This shows that $\delta^{2}=0$. The homology of this complex, known as the cyclic Hochschild cohomology, is denoted by $H C^{\bullet}(A)$.

4.2. PROP $\mathcal{D} \mathcal{G}_{k}^{\bullet}$ of directed graphs of type $k$. One basic idea is that an incoming edge can connect to an outgoing edge, as indicated in Figure 15. We are interested in graphs that are obtained by connecting vertices of mixed types.

Definition 4.1. $\mathcal{D} \mathcal{G}_{\infty}^{\bullet}$ is the vector space generated by all pairs $(\gamma, \mathcal{O})$, where $\gamma$ is a directed graph with a cyclic ordering of the edges at each vertex of type $0,1,2, \cdots$, with the proviso that type 1 vertices have at least two incoming edges, and $\mathcal{O}$ is an orientation on the graded vector space generated by the edges and vertices of type $n \neq 0$ of $\gamma$, where the degree of an edge is 1 , and the degree of a vertex of type $n$ is $n(2-d)+(d-4)$.

Furthermore, we define $\mathcal{D G}_{k}^{\bullet}$, for $k=1,2,3 \cdots$, to be the subspace of $\mathcal{D} \mathcal{G}_{\infty}^{\bullet}$ given by graphs with vertices of type $n$ where $n \leq k$.

Figure [16] shows an example of a graph in $\mathcal{D G}_{\infty}^{\bullet}$. For each such graphs, we have so called inputs and outputs. By definition, the inputs are the vertices of type 0 . The outputs of the graph are the boundaries of the thickened (fat) graph. See for example Figure [17 where the three outputs are indicated by dotted, dashed, and thin solid curves. Both inputs and outputs are enumerated as part of the structure. Note that there may be no input, but there has to be at least one output.

The grading in $\mathcal{D} \mathcal{G}_{\infty}^{\bullet}$ is given in the following way. Look at a graph in $\mathcal{D} \mathcal{G}_{\infty}^{\bullet}$. Every nontrivial vertex of type 0 with $m$ incoming edges contributes $m-1$ to the degree, whereas vertices of type $n \geq 1$ with $m$ 


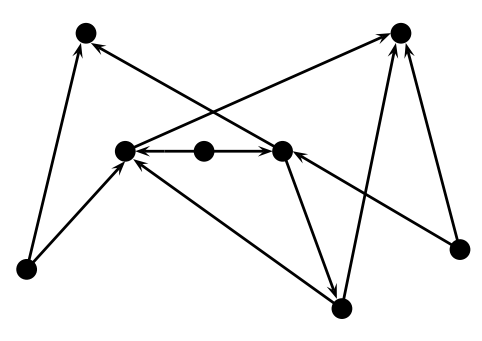

FiguRE 16. A combinatorial directed graph

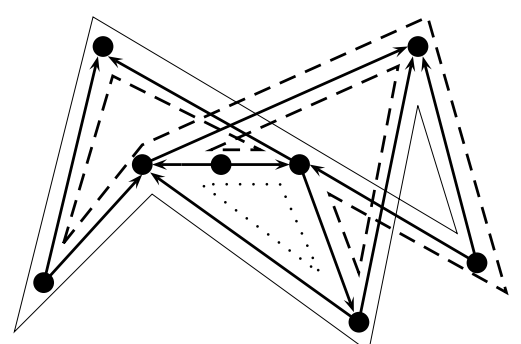

FiguRE 17. 2 inputs and 3 outputs

incoming edges add $2 n+m-2$ to the degree. Trivial vertices have degree zero. This introduces a grading $\mathcal{D} \mathcal{G}_{\infty}^{\bullet}=\bigoplus_{n \in \mathbb{N} \cup\{0\}} \mathcal{D} \mathcal{G}_{\infty}^{n}$. Note that none of the graphs have negative degree in $\mathcal{D G}_{\infty}^{\bullet}$, since we restricted type 1 vertices to have at least two incoming edges. In fact, the lowest degree $\mathcal{D} \mathcal{G}_{\infty}^{0}$ consists of type 0 vertices with zero or one incoming edge, type 1 vertices with two incoming edges, and type 2 vertices with no incoming edges; see Figure 18. Thus, the lowest operations are given by the product and the co-inner product, whereas all higher vertices, including all vertices of type $\geq 3$, are interpreted as homotopies.

$\mathcal{D} \mathcal{G}_{\infty}^{\bullet}$ is in fact a complex. The differential $D: \mathcal{D G}_{\infty}^{\bullet} \rightarrow \mathcal{D G}_{\infty}^{\bullet-1}$ is defined by expansion of an edge. To be more precise, $D$ applied to a graph is defined to be the sum of all permitted directed graphs that preserve the number of type 0 vertices, such that collapsing an edge produces the original graph. An example is shown in Figure 19, where the first three graphs of the boundary expand the lower left type 3 vertex, the next two graphs expand the upper two vertices of type 0 , and the last two graphs expand the lower right vertex of type 2 . The orientations of the resulting graphs, which we suppressed in Figure 19. 


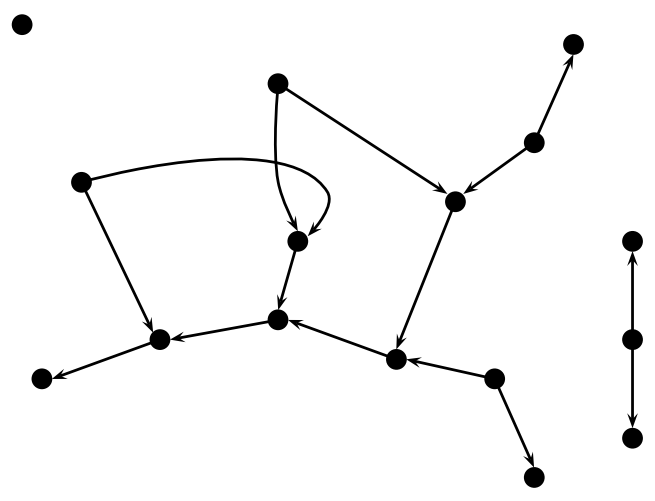

Figure 18. A graph in $\mathcal{D} \mathcal{G}_{\infty}^{0}$

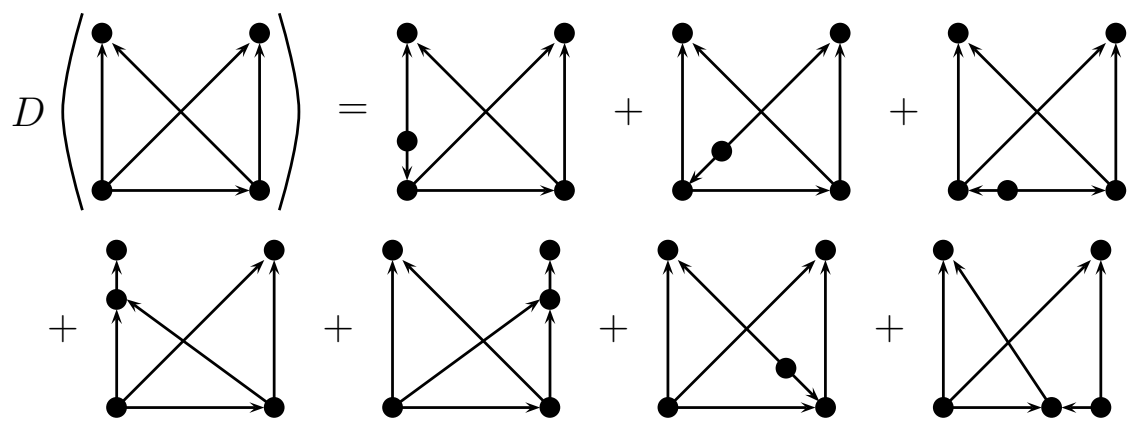

Figure 19. Boundary of an element in $\mathcal{D G}_{\infty}^{\bullet}$

are obtained by bringing the relevant vertex in the expression determining the orientation to the front, and replacing it by the added edge followed by the two vertices in the order given by the direction of the expanded edge. Note that $D^{2}=0$, since $D^{2}$ expands two edges, which can be done in two ways, depending on the ordering of the expanded edges.

We next explain how to compose two graphs. Suppose $\gamma_{1}$ is a graph with $i$ inputs and $j$ outputs, and let $\gamma_{2}$ be a graph with $j$ inputs and $k$ outputs. The composition will then be a graph $\gamma_{1} \circ \gamma_{2}$ with $i$ inputs and $k$ outputs. Identify the outputs of $\gamma_{1}$ with the inputs of $\gamma_{2}$. Then, starting with the graph $\gamma_{1}$, we perform the operation from Figure 20 at each output of $\gamma_{1}$. More precisely, we take the edges at an input of $\gamma_{2}$ and attach them to the corresponding output of $\gamma_{1}$ in all possible ways. The orientation of the composed graph $\gamma_{1} \circ \gamma_{2}$ is given by attaching the orientation of $\gamma_{2}$ to that of $\gamma_{1}$ on the right. Note that if 


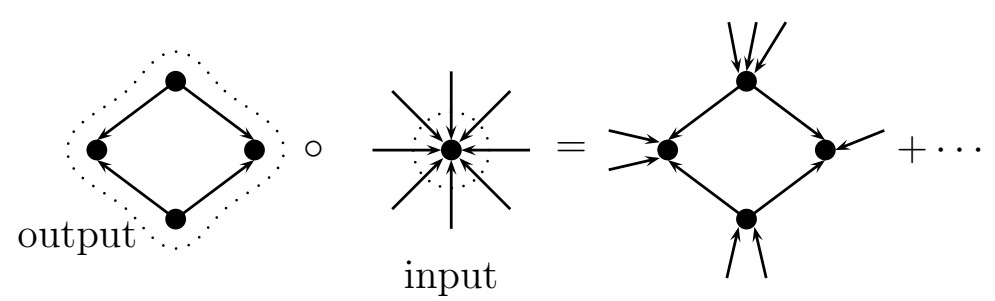

Figure 20. The composition

we thicken the graphs to a surface and punch holes at locations of the type zero vertices, the composition corresponds exactly to the gluing of the surfaces along $j$ boundary components. This picture also shows that the composed graph has in fact exactly $k$ outputs.

The above definitions give $\mathcal{D} \mathcal{G}_{\infty}^{\bullet}$ and each $\mathcal{D} \mathcal{G}_{k}^{\bullet}$ the structure of a PROP. Note that there are obvious inclusion maps $\mathcal{D G}_{k}^{\bullet} \hookrightarrow \mathcal{D} \mathcal{G}_{k+1}^{\bullet}$ and $\mathcal{D G}_{k}^{\bullet} \hookrightarrow \mathcal{D} \mathcal{G}_{\infty}^{\bullet}$, for every $k=1,2, \cdots$.

Proposition 4.2. There is a sequence of inclusions of PROPs,

$$
\mathcal{D G}_{1}^{\bullet} \hookrightarrow \mathcal{D} \mathcal{G}_{2}^{\bullet} \hookrightarrow \mathcal{D} \mathcal{G}_{3}^{\bullet} \hookrightarrow \cdots \hookrightarrow \mathcal{D} \mathcal{G}_{\infty}^{\bullet} .
$$

4.3. Algebraic structure of the cyclic Hochschild complex. We are now prepared to describe how, for an $\mathrm{V}_{\infty}$-algebra $A$, the PROP $\mathcal{D} \mathcal{G}_{\infty}^{\bullet}$ acts on $C C^{\bullet}(A)$.

Theorem 4.3. Let $k=1,2, \cdots, \infty$, and let $A$ be a $V_{k}$-algebra. Then, there is a map,

$$
\mathcal{D} \mathcal{G}_{k}^{\bullet} \rightarrow \prod_{r, s} \operatorname{Hom}\left(C C^{\bullet}(A)^{\otimes r}, C C^{\bullet}(A)^{\otimes s}\right),
$$

which respects the grading, differential, and composition.

Here is a sketch of the proof for $\mathcal{D} \mathcal{G}_{\infty}^{\bullet}$. The proof for $\mathcal{D} \mathcal{G}_{k}^{\bullet}$ is similar. For any graph in $\mathcal{D} \mathcal{G}_{\infty}^{\bullet}$ with $r$ inputs and $s$ outputs, we will obtain a mapping $C C^{\bullet}(A)^{\otimes r} \rightarrow C C^{\bullet}(A)^{\otimes s}$. The elements $f_{1}, \cdots, f_{r} \in C C^{\bullet}(A)$ are to be plugged into the type 0 vertices enumerated by $1, \cdots, r$. In detail, we consider the $j^{\text {th }}$ vertex of type 0 , together with all possible hairs, as indicated in Figure 21] If $f_{j}$ is given by a sum of terms $a_{1}^{*} \otimes \cdots \otimes a_{n}^{*}$, then choose one of the edges as your initial edge, and apply $a_{1}^{*}, \cdots, a_{n}^{*}$ cyclically at this vertex. We shall see that the final outcome will be independent of the choice of the starting point; see section 4.1 Note that the total number of edges and hairs must add up exactly to $n$, the number of the tensor factors. If there are fewer than $n$ edges, then add an appropriate number of hairs in all possible 


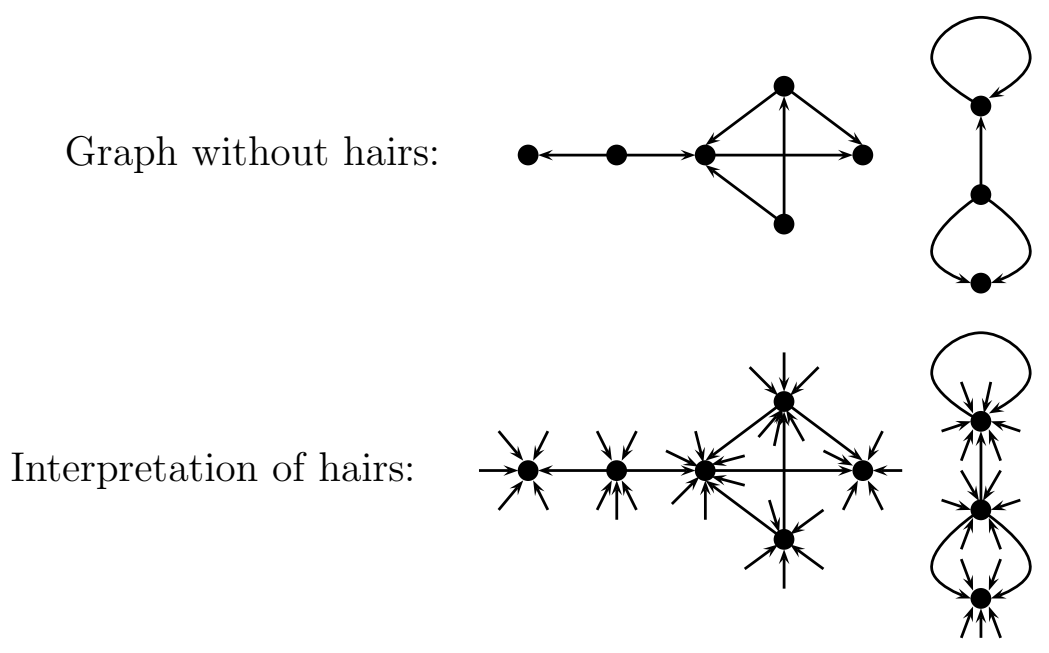

FiguRE 21. Graphs interpreted with hairs

ways. If there are more than $n$ edges, then $a_{1}^{*}, \cdots, a_{n}^{*}$ cannot be applied to the vertex and would contribute a zero to the sum.

Next, we also apply the $\mathrm{V}_{\infty}$-algebra structure to the vertices of type $k \geq 1$. Just as with vertices of type 0 , we always add the appropriate number of hairs and sum over all possible ways. Since type 1 vertices have a unique outgoing edge, the application of elements of $A$ and $A^{*}$ is given without ambiguity. Similarly, we assign the element $v_{i_{1}, \cdots, i_{k}}$ of the $\mathrm{V}_{\infty}$-algebra structure to a vertices of type $k$. The symmetry condition from Definition 3.1] guarantees that this application is, up to a sign, independent of choice of first outgoing edge; see Figure 8. There is an overall sign determined by the orientation $\mathcal{O}$ which fixes this ambiguity in sign.

After applying these algebraic data to all vertices, using the paring between $A^{*}$ and $A$, the dual elements on each of the edges are contracted so that the only remaining terms are the hairs. We can now read off the outputs along the outputs of the graph. These outputs have no starting points and need to be symmetrized cyclically to become elements of $C C^{\bullet}(A)$.

Note that the above procedure is only well defined element up to sign. For one, the symmetry condition determines $v_{i_{1}, \cdots, i_{k}}$ at a vertex only up to sign, and a second problem arises when contracting $A^{*}$ with $A$, since we shifted $A^{*}$, but not $A$. These ambiguities may be resolved, using the orientation on the vertices and edges of the graph in $\mathcal{D} \mathcal{G}_{\infty}^{\bullet}$. In fact, using the orientation, as well as the cyclic ordering of the edges at each vertex, we may linearize the expression for all vertices 
and put the inverse of the shift operators in those places where edges appear. The inverse of the shift operators, one for each edge in the expression for the orientation, will be used to shift back elements of $A^{*}$ before evaluating them on elements of $A$. The remaining terms in the linearization may be rearranged in accordance with the rules dictated by the graph. The symmetry condition guarantees a well defined overall sign for the outcome of the operation after the rearrangement, since we only made repeated use of the Koszul rule.

In order to check that the differentials are preserved, recall that the differential on $C C^{\bullet}(A)$ is given by applying one additional $\mathrm{A}_{\infty}$-algebra map (type 1 vertex) as described in Figure [14. The differential in the space of operations $\operatorname{Hom}\left(C C^{\bullet}(A)^{\otimes r}, C^{\bullet}(A)^{\otimes s}\right)$ applies this differential to all inputs and outputs. This cancels all additional $\mathrm{A}_{\infty}$-algebra maps on hairs of type 0 vertices, and adds a type 1 vertex at the type 0 vertices in all possible ways. The remaining additional $A_{\infty}$-algebra maps together with the boundary condition from Definition 3.1 shows that, in fact, one has to add one vertex at any possible position of the graph. This coincides with the differential in $\mathcal{D} \mathcal{G}_{\infty}^{\bullet}$.

Finally, we deal with the compositions. Consider the usual composition of $\operatorname{Hom}\left(C C^{\bullet}(A)^{\otimes r}, C C^{\bullet}(A)^{\otimes s}\right)$ and $\operatorname{Hom}\left(C C^{\bullet}(A)^{\otimes s}, C C^{\bullet}(A)^{\otimes t}\right)$ with output in $\operatorname{Hom}\left(C C^{\bullet}(A)^{\otimes r}, C C^{\bullet}(A)^{\otimes t}\right)$. In terms of graphs in $\mathcal{D} \mathcal{G}_{\infty}^{\bullet}$, this means that the $s$ outputs of the first graph have to be taken as the inputs for the second graph. We defined the $s$ outputs as the leftover hairs after applying the edges of the graph. Thus, we need to apply the edges at the inputs (type 0 vertices) of the second graph, to the outputs of the first graph in all possible ways permitted by the combinatorics of the $s$ outputs. This is exactly the definition of the composition as explained in Figure 20.

4.4. An example: associative algebras with co-inner products. Recall that a differential graded associative algebra with an invariant and symmetric co-inner product is an examples of a $\mathrm{V}_{\infty}$-algebra. In fact, in this case only the lowest maps $v_{2}=\mu_{2}$ and $v_{0,0}=U$ are non zero. That is to say, $v_{i}$ 's for $i \neq 2, v_{\left(i_{1}, i_{2}\right)}$ 's for $\left(i_{1}, i_{2}\right) \neq(0,0)$, and $v_{i_{1}, \cdots, i_{k}}$ 's for $k \geq 3$ are all zero. In this section, we want to show that in this special case the action of $\mathcal{D G}_{\infty}^{\bullet}$ reduces to the action of the cyclic Sullivan chord diagrams without marked points, as described in section 2.2 .

Most of the $v_{i_{1}, \cdots, i_{k}}$ 's vanish, and the only non-zero graphs in $\mathcal{D} \mathcal{G}_{\infty}^{\bullet}$ have vertices of types 1 and 2 in the lowest degree. Furthermore, note that due to associativity and bimodule conditions in Figure 5 and Figure 7 , one can slide type 1 vertices along any type 2 vertices and also 

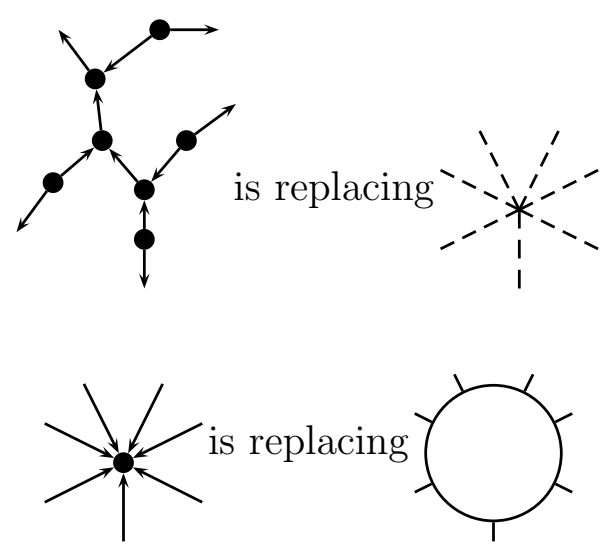

Figure 22. Chords and strings in the strict case

along other type 1 vertices. By slight abuse of language, we now call the edges together with vertices of type 1 and 2 , the chords of the graph. This makes sense, since by the above remark, the exact order in which type 1 and 2 vertices are applied is irrelevant, and a chord is specified by only knowing the cyclic combinatorics of its attached type 0 vertices. We will represent chords by dashed lines and vertices of type 0 by open circles, as in Figure 22, Non-vanishing graphs in $\mathcal{D} \mathcal{G}_{\infty}^{\bullet}$ will therefore consist of circles with chords attached; see Figure 3 .

Finally, an orientation for a graph reduces to an orientation as described in section 2.2, since, for $d=0$, all the vertices in the graph are of even degree, and shifts in a chord may be canonically arranged, so that they only apply to the chords endpoints.

Our graphs now become just as described in section 2.2. By the Theorem 4.3 from section 4.3 graphs without marked points act on the cyclically invariant part of the Hochschild complex. This implies, for example, a Lie algebra structure on the cyclic Hochschild cohomology, which was also noticed by L. Menichi in [M].

4.5. Obstructions and operations. In this section, we want to describe how the higher type vertices are used to show that the cyclic Hochschild cohomology of a $V_{k}$-algebra, $k \geq 3$, is a Lie algebra. Notice that every $V_{2}$-algebra $A$ induces an operation $[\cdot, \cdot]: C C^{\bullet}(A) \otimes$ $C C^{\bullet}(A) \rightarrow C C^{\bullet}(A)$. We choose the orientation $v \wedge e_{1} \wedge e_{2}$ on the vectors space generated by the (type 2 ) vertex and the two edges, so that reversing the inputs shows that we have a skew-symmetric operation. This bracket is closed, and therefore descends to the cyclic Hochschild cohomology $H C^{\bullet}(A)$. It turns out that this bracket, in general, does 


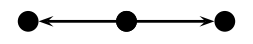

Figure 23. The bracket $[\cdot, \cdot]$

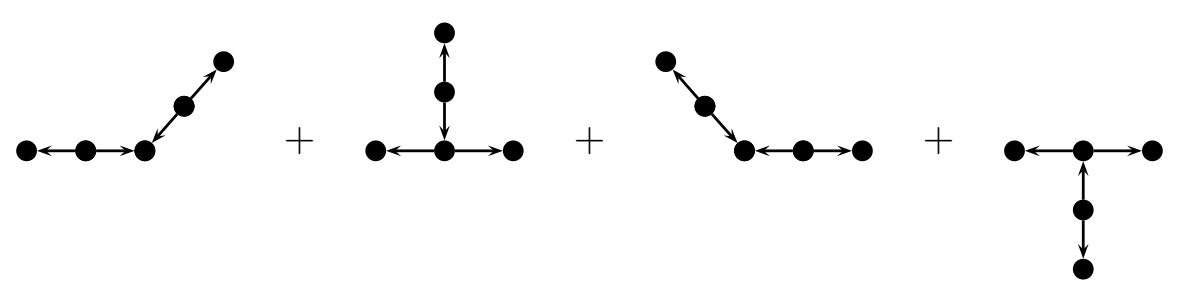

Figure 24. The term $[[\cdot, \cdot], \cdot]$ for the Jacobi identity

not satisfy Jacobi identity. However, in the case of a $V_{3}$-algebra, the vertices of type 3 provide homotopies for the Jacobi identity.

Proposition 4.4. Let $A$ be a $V_{3}$-algebra and $[\cdot, \cdot]: C C^{\bullet}(A) \otimes C C^{\bullet}(A) \rightarrow$ $C C^{\bullet}(A)$ denote the operation labelled by the graph in Figure 23. Then, $[\cdot, \cdot]$ is skew symmetric and satisfies the Jacobi identity up to homotopy.

Proof. In order to see that the Jacobi identity holds, we need to compare $[[\cdot, \cdot], \cdot]$ with its two cyclic rotations. The graphs for the term $[[\cdot, \cdot], \cdot]$ of the Jacobi identity are shown in Figure 24. The first and third terms only differ by their enumeration of their three inputs. As required by the Jacobi identity, we also obtain graphs where these enumerations are cyclically rotated. A comparison of their orientations shows the cancellation of these six graphs in the Jacobi identity.

The remaining second and fourth term of $[[\cdot, \cdot], \cdot]$ together with their cyclically rotated input enumerations are exactly the terms of the boundary of the type 3 vertex; see Figure 25.

\section{SOME VARIATIONS}

We now consider several variations of the discussion of the previous section. In particular, we will consider loops (Hochschild complex) and open strings (two-sided cobar complex). Finally, in section 5.3. we give a topological picture which might lend insight to some of our constructions.

5.1. Open/closed string operations for $\mathbf{V}_{\infty}$-algebras. So far, we have only considered the case of the cyclic Hochschild complex, or the closed strings. It is natural to extend this to the cyclic Hochschild 


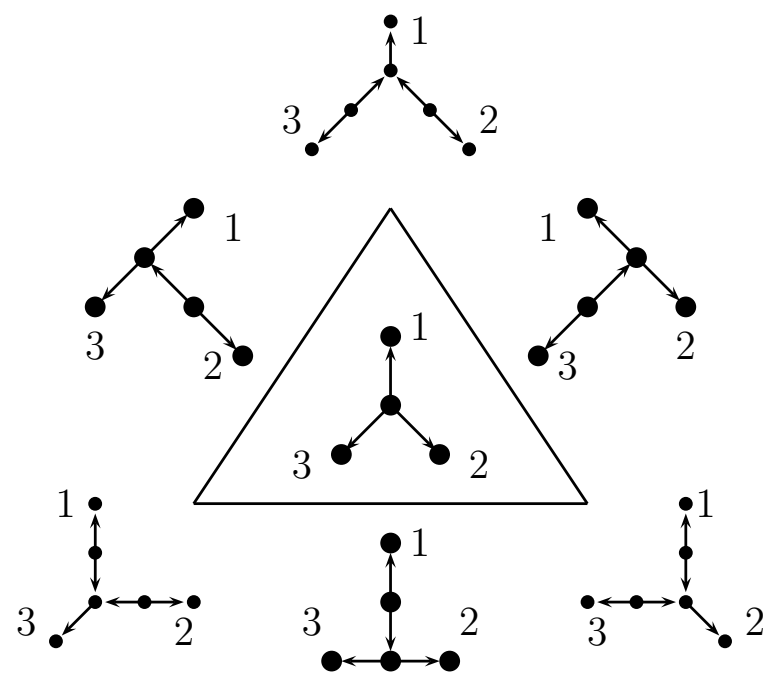

Figure 25. The homotopy induced by the vertex of type 3
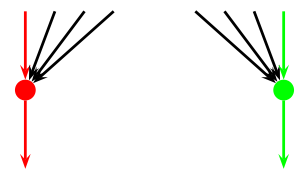

Figure 26. Homotopy left and right module structures

complex coupled with a two-sided cobar complex, or an open/closed version of the string topology. The interaction of open and closed strings is of considerable interest (see for example, [BCR, [C], [H], KaSt], or [Su] ).

We now show how to extend our discussion to the open case. Let us start with the generalizations of the concepts of left and right modules. Let $A$ be a differential graded associative algebra, and let $M$ and $N$ be differential graded left and right modules, respectively. In other words, there are chain maps $M \otimes A \rightarrow M$, and $A \otimes N \rightarrow N$, respecting the product in $A$. The homotopy versions require sequences of maps $\left\{\lambda_{n}: M \otimes A^{\otimes n} \rightarrow M\right\}_{n \geq 0}$, and $\left\{\rho_{n}: A^{\otimes n} \otimes N \rightarrow N\right\}_{n \geq 0}$, where $\lambda_{0}$ and $\rho_{0}$ are the differentials $\partial_{M}$ and $\partial_{N}$ of $M$ and $N$, respectively. Graphically, we will distinguish $A, M$ and $N$ by assigning the colors black, red, and green to $A, M$ and $N$, respectively. Then, the left and right module structures are shown as in Figure 26. and the required relations are as indicated in Figure 27. 


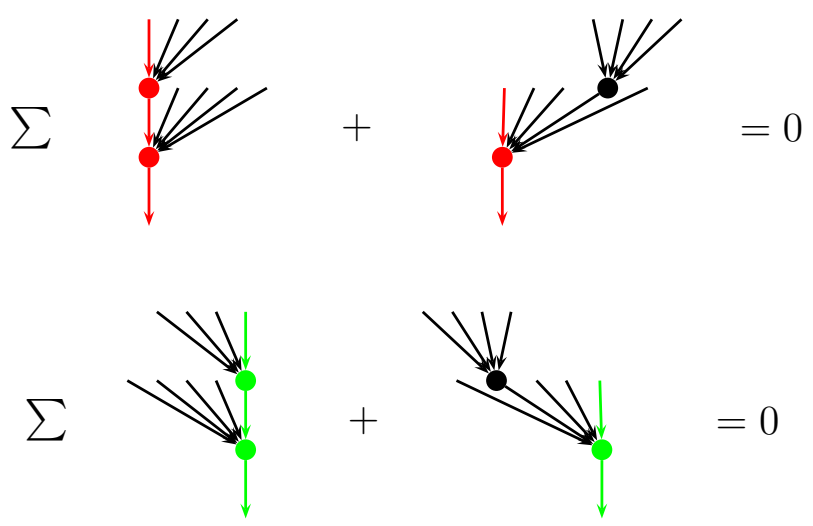

FiguRE 27. Homotopy left and right module relations

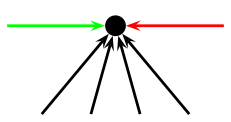

Figure 28. The cobar complex $C^{\bullet}(M, A, N)$

Next, look at the two-sided cobar complex,

$$
C^{\bullet}(M, A, N)=\prod_{j=0}^{\infty}\left\{f: M \otimes A^{\otimes j} \otimes N \rightarrow \mathbf{k}\right\} .
$$

The differential $\delta: C^{\bullet}(M, A, N) \rightarrow C^{\bullet}(M, A, N)$ is given by,

$$
\begin{aligned}
\delta f\left(m, a_{1}, \cdots, a_{j}, n\right)= & \sum_{l} \pm f\left(\lambda_{l}\left(m, a_{1}, \cdots, a_{l}\right), \cdots, a_{j}, n\right) \\
+ & \sum_{l, i} \pm f\left(m, a_{1}, \cdots, \mu_{l}\left(a_{i}, \cdots, a_{i+l-1}\right), \cdots, a_{j}, n\right) \\
+ & \sum_{l} \pm f\left(m, a_{1}, \cdots, \rho_{l}\left(a_{j-l+1}, \cdots, a_{j}, n\right)\right) .
\end{aligned}
$$

Once again, the signs are again given by comparing the linear order of the symbols in an expression to the orientation $\psi \wedge f \wedge m \wedge a_{1} \wedge \cdots \wedge a_{j} \wedge n$, where $\psi=\mu_{l}, \lambda_{l}$, or $\rho_{l}$, is of degree 1 , and $m, n$, as well as the $a_{i}$ 's, have shifted degrees. The elements of $C^{\bullet}(M, A, N)$ will be represented by vertices with $j$ black incoming edges together with one red and one green incoming edge on the left and right, respectively; see Figure 28.

We want to consider all graphs, consisting of vertices of the above type. We also need to add red and green edges with only one outgoing edge and no incoming edges, corresponding to the identity map $M \rightarrow$ 


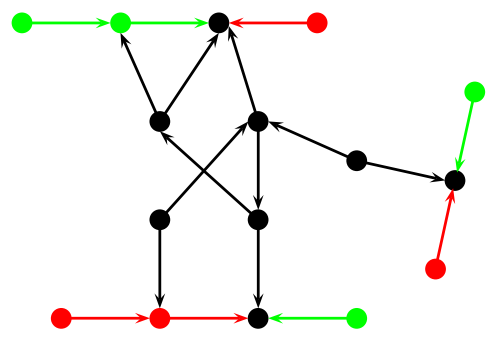

FiguRE 29. An open/closed graph

$M$ or $N \rightarrow N$, respectively. In this manner, we obtain graphs similar to that of Figure 29.

Let $\operatorname{col} \mathcal{D G}_{\infty}^{\bullet}$ denote the space of graphs that include the above colored vertices, with orientations given by all vertices and all edges of type $n \neq 0$, where the red and green vertices from Figure 26 have degree -2 . Furthermore, $\operatorname{colD}_{\infty} \mathcal{G}_{\infty}^{\bullet}$ has a differential and a composition, which are defined similarly to those in section 4.2. With these adjustments, we can extend the theorem from section 4.3 in the following way.

Theorem 5.1. There is a map,

$$
\begin{aligned}
\operatorname{colD} \mathcal{G}_{\infty}^{\bullet} \rightarrow & \prod_{r_{c}, r_{o}, s_{c}, s_{o}} \operatorname{Hom}\left(C C^{\bullet}(A)^{\otimes r_{c}} \otimes C^{\bullet}(M, A, N)^{\otimes r_{o}},\right. \\
& \left.C C^{\bullet}(A)^{\otimes s_{c}} \otimes C^{\bullet}(M, A, N)^{\otimes s_{o}}\right),
\end{aligned}
$$

which respects the grading, differential, and composition.

Remark 5.2. The above theorem implies that $H^{\bullet}(M, A, N)$, the homology of the complex $C^{\bullet}(M, A, N)$, is a module over $H C^{\bullet}(A)$. In section 5.3, we will see how $M$ and $N$ correspond to subcomplexes of a given manifold; see Figure 35. The module structure described in this section corresponds to the intersection of cells inside the big manifold.

But, this is only half the story. When the subcomplexes are in fact submanifolds, then they will also have Poincaré duality, making it possible for intersection within them. Algebraically, we might add this structure by requiring $M$ and $N$ to also have (colored) homotopy coinner products. This would give a further extension of the PROP $\operatorname{col} \mathcal{D G}_{\infty}^{\bullet}$, with a similar action as in Theorem 5.1 .

5.2. Algebraic structure of the Hochschild complex. In this section, we consider the Hochschild complex $C H^{\bullet}\left(A, A^{*}\right)$. A modification 


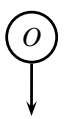

FiguRE 30. The output marked point

of the string PROP, that corresponds to introducing marked points, will give the desired PROP action in the non-cyclic setting.

Let $A$ be a $\mathrm{V}_{\infty}$-algebra. An element $\mathbf{1} \in A$ is called a strict unit, or simply a unit, if it satisfies the relations,

$$
\begin{aligned}
v_{2}(a, \mathbf{1})=v_{2}(\mathbf{1}, a)=a & \text { for } a \in A \\
v_{i_{1}, \cdots, i_{k}}(\cdots, \mathbf{1}, \cdots)=0 & \text { for all other }\left(i_{1}, \cdots, i_{k}\right) \neq(2) .
\end{aligned}
$$

The Hochschild cochain complex of $A$ with values in the dual space $A^{*}$ is given by,

$$
C H^{\bullet}\left(A, A^{*}\right)=\prod_{j=0}^{\infty}\left\{f: A^{\otimes j} \rightarrow A^{*}\right\} .
$$

In the presence of a unit, the normalized subcomplex is defined by,

$$
\begin{aligned}
\overline{C H^{\bullet}}\left(A, A^{*}\right) & =\prod_{j=0}^{\infty}\left\{f: A^{\otimes j} \rightarrow A^{*} \mid\right. \\
& \left.f\left(a_{1}, \cdots, a_{j}\right)=0 \text {, if any of the } a_{1}, \cdots, a_{j} \text { equals } \mathbf{1}\right\} .
\end{aligned}
$$

The induced differential on this subspace makes it into a subcomplex, which is quasi-isomorphic to the big complex via the inclusion $\overline{C H^{\bullet}}\left(A, A^{*}\right) \hookrightarrow C H^{\bullet}\left(A, A^{*}\right)$; see $[\mathrm{L}$. In what follows, we identify a map $A^{\otimes j} \rightarrow A^{*}$ with a map $A^{\otimes j+1} \rightarrow \mathbf{k}$.

We now define a new graph complex, $m \mathcal{D} \mathcal{G}_{\infty}^{\bullet}$, suitable for treating the normalized Hochschild complex. $m \mathcal{D} \mathcal{G}_{\infty}^{\bullet}$ is a variation of $\mathcal{D} \mathcal{G}_{\infty}^{\bullet}$ that deals with the issues of starting points for inputs and outputs, as well as the unit. Here are what we need,

(1) We need marked point at every vertex of type 0. By definition, a marked point of an input is a choice of edge attached to that input. For the trivial type 0 vertex without edges, no choice is necessary. We will denote this chosen edge by a double-headed arrow; see Figure 32

(2) A marked point for an output consists of exactly one additional external leg for each output. We denote this leg by a vertex with one outgoing edge and no incoming edges; see Figure 30. 


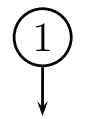

Figure 31. The unit vertex

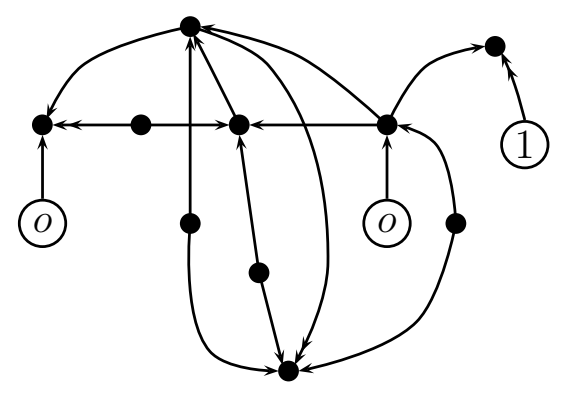

Figure 32 . A graph in $m \mathcal{D} \mathcal{G}_{\infty}^{\bullet}$

(3) The unit $\mathbf{1} \in A$ gives rise to a new vertex with exactly one outgoing edge and no incoming edges; see Figure 31. By the defining relations of the unit, we may never attach the unit to any $v_{i_{1}, \cdots, i_{k}}$, except for $v_{i}$, when $i=2$, for which we may ignore the unit, since $v_{2}(a, \mathbf{1})=v_{2}(\mathbf{1}, a)=a$. Thus, the unit may only be applied to vertices of type 0 . Furthermore, since we work with the normalized Hochschild complex, the unit can only be applied in the last slot $f(\cdots, \mathbf{1})$ of any $f \in \overline{H C \bullet}\left(A, A^{*}\right)$. This means that if the unit vertex occurs, it necessarily has to be the marked point of a type 0 vertex.

An example of a graph in $m \mathcal{D G}_{\infty}^{\bullet}$ is shown in Figure 32. The differential on $m \mathcal{D} \mathcal{G}_{\infty}^{\bullet}$ is again given by expansion of an edge, where we now have to preserve the marked points of the inputs and outputs. Similarly, when composing the graphs $\gamma_{1}$ and $\gamma_{2}$, we also need to match the marked edges. This means that when attaching the edges from some input of $\gamma_{2}$ to the corresponding output of $\gamma_{1}$, the marked edge of the input has to coincide with that of the output. With this, we have the following theorem.

Theorem 5.3. There is a map,

$$
m \mathcal{D} \mathcal{G}_{\infty}^{\bullet} \rightarrow \prod_{r, s} \operatorname{Hom}\left(\overline{H C} \bullet\left(A, A^{*}\right)^{\otimes r}, \overline{H C^{\bullet}}\left(A, A^{*}\right)^{\otimes s}\right)
$$

which respects the grading, differential, and composition.

The following comments are in order. 
Remark 5.4. Let $1 \in A$ denote the unit. We can extend the definition of a $\mathrm{V}_{\infty}$-algebra $\left\{v_{i_{1}, \cdots, i_{k}}\right\}_{k}$ satisfying all the relations from Definition 3.1 by allowing for $v_{0}$ to exist; see Remark 3.2. Here, the element $v_{0} \in A$ will be called a weak unit which makes $A$ into a weak $\mathrm{A}_{\infty^{-}}$ algebra.

Remark 5.5. In the case of strictly associative algebras, with the help of the enumeration of the inputs and the linear ordering of the marked points on the input circles, the orientation on vertices and edges may be chosen canonically. Thus, in this case, our constructions and results reduce to those of section 2.1.

Remark 5.6. The graph complex $\mathcal{D G}_{\infty}^{\bullet}$ is reminiscent of that considered in KoSo] by Kontsevich and Soibelman. They define an operad $M$ that naturally acts on the Hochschild cochain complex $C H^{\bullet}(A, A)=$ $\prod_{j=0}^{\infty}\left\{f: A^{\otimes j} \rightarrow A\right\}$ of an $\mathrm{A}_{\infty}$-algebra $A$. The operad $M$ is made out of trees that have only type 1 vertices which are used for both the components of the $\mathrm{A}_{\infty}$-algebra structure as well as for labelling the elements of $C H^{\bullet}(A, A)$.

Their discussion in KoSo goes further by identifying $M$ with the Strebel differentials on the Riemann sphere. Thus, a quasi-isomorphism between the operad $M$ and the chains on the little disc operad is established, which in turn solves the Deligne conjecture. We suspect a strong relationship between $\mathcal{D G}_{\infty}^{\bullet}$ and the chains on a moduli space of Riemann surfaces.

Remark 5.7. This work is about the associative operad. It is reasonable to expect that similar results would hold for any cyclic operad $\mathcal{O}$. More precisely, we expect that the space of directed graphs with an $\mathcal{O}$ cyclic order on their vertices would act on the cyclic cochain complex associated to the operad $\mathcal{O}$. See [LT] for a study of homotopy co-inner products on cyclic operads.

5.3. Topological applications and motivations. Let $X$ denote a simply connected and triangulated homology manifold of dimension $d$. In a previous work [TZ1, we showed that the (simplicial) cochains $C^{\bullet} X$ form a $\mathrm{V}_{2}$-algebra, i.e., an $\mathrm{A}_{\infty}$-algebra with an invariant and symmetric homotopy co-inner product. This was achieved by obtaining an invariant and symmetric homotopy inner product that was moreover nondegenerate in an appropriate sense. It was then argued, using minimal models, how this nondegeneracy gives rise to an invariant and symmetric homotopy co-inner product. Note that in this paper coinner products do not satisfy any nondegeneracy assumptions. An immediate application of Theorem 4.3 is the following corollary. 


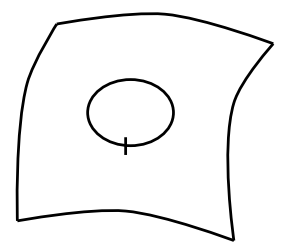

Figure 33. A loop in a manifold

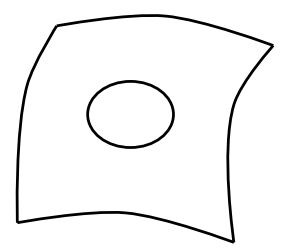

Figure 34. A closed string in a manifold

Corollary 5.8. The PROP $\mathcal{D G}_{2}$ acts on the cyclic Hochschild complex $C C^{\bullet}\left(C^{\bullet} X\right)$.

Corollary 5.9. The PROP $m \mathcal{D G}_{2}$ acts on the Hochschild complex $C H^{\bullet}\left(C^{\bullet} X\right)$.

It is known that for a simply connected $X$, the Hochschild complex $C H^{\bullet}\left(C^{\bullet} X, C \bullet X\right)$ and the cyclic Hochschild complex $C C^{\bullet}\left(C^{\bullet} X\right)$ are quasi-isomorphic to the chains on the free loop space, $C_{\bullet} L X$, and the equivariant chains on the free loop space, $C_{\bullet}^{S^{1}} L X$, respectively; see Figure 33 and Figure 34. Therefore, we have,

Corollary 5.10. The PROP $\mathcal{D} \mathcal{G}_{2}$ acts on $C_{\bullet}^{S^{1}} L X$.

Corollary 5.11. The PROP $m \mathcal{D G}_{2}$ acts on $C . L X$.

Corollary 5.12. $H_{\bullet} L X$ is, after an appropriate shift in degrees, a $B V$ algebra.

Also, for simplicial subcomplexes $K, L \subset X$, let $P(K, X, L)$ be the space of paths in $X$ starting in $K$ and ending in $L$. The cochains $C^{\bullet} K$ and $C^{\bullet} L$ are left and right modules over $C^{\bullet} X$, and the two-sided cobar complex $C^{\bullet}\left(C^{\bullet} K, C^{\bullet} X, C^{\bullet} L\right)$ is naturally quasi-isomorphic to the chains of the path space $C_{\bullet}(P(K, X, L))$; see Figure 35.

We believe there is a deeper connection between string topology and the structures discussed in this paper. More specifically, we expect that for a triangulated Poincaré duality space $X$, the simplicial chains $C^{\bullet} X$ to be a $\mathrm{V}_{\infty}$-algebra, which in turn would imply that $\mathcal{D} \mathcal{G}_{\infty}^{\bullet}, m \mathcal{D} \mathcal{G}_{\infty}^{\bullet}$, 


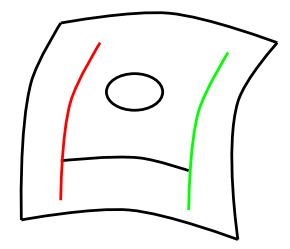

FiguRE 35. Open/closed strings in a manifold

and $\operatorname{colDS}_{\infty}^{\bullet}$ act on $C C^{\bullet}\left(C^{\bullet} X\right), C H^{\bullet}\left(C^{\bullet} X, C \cdot X\right)$, and $C C^{\bullet}\left(C^{\bullet} X\right) \otimes$ $C^{\bullet}\left(C^{\bullet} K, C^{\bullet} X, C^{\bullet} L\right)$, respectively.

In light of all this, one also expects an action of $\mathcal{D G}_{\infty}^{\bullet}$ on $C_{\bullet}^{S^{1}} L X$, an action of $m \mathcal{D} \mathcal{G}_{\infty}^{\bullet}$ on $C_{\bullet} L X$, and an action of $\operatorname{col} \mathcal{B} \mathcal{G}_{\infty}^{\bullet}$ on $C_{\bullet}^{S^{1}} L X \otimes$ $C_{\bullet}(P(K, X, L))$. It is highly desirable to understand the relationship between the PROP $\mathcal{D} \mathcal{G}_{\infty}^{\bullet}$ and the PROP of the chains on DeligneMumford compactification of the moduli space of Riemann surfaces.

\section{REFERENCES}

[BCR] N.A. Baas, R.L. Cohen, A. Ramirez, The topology of the category of open and closed strings, AT/0411080, (2004)

[CS1] M. Chas, D. Sullivan, String Topology, GT/9911159, (1999)

[CS2] M. Chas, D. Sullivan, Closed string operators in topology leading to Lie bialgebras and higher string algebra, The legacy of Niels Henrik Abel, 771784, Springer, Berlin, (2004)

[C] K. Costello, Topological conformal field theories and Calabi-Yau categories, QA/0412149, (2004)

[G] M. Gerstenhaber, The cohomology structure of an associative ring, Annals of Mathematics, Vol. 78, No. 2, (1963)

[H] E. Harrelson, On the homology of open-closed string theory, AT/0412249, (2004)

[Kaj] H. Kajiura, Noncommutative homotopy algebras associated with open strings, QA/0306332, (2003)

[KaSt] H. Kajiura, J. Stasheff, Homotopy algebras inspired by classical open-closed string field theory, QA/0410291, (2004)

[Kau] R.M. Kaufmann, On spineless cacti, Deligne's conjecture and ConnesKreimer's Hopf algebra, QA/0308005, (2003)

[KoSo] M. Kontsevich, Y. Soibelman, Deformations of algebras over operads and Deligne's conjecture, Confrence Mosh Flato 1999, Vol. I (Dijon), 255-307, Math. Phys. Stud., 21, Kluwer Acad. Publ., Dordrecht, (2000)

[L] J.-L. Loday, Cyclic Homology, Grundlehren der mathematischen Wissenschaften 301, Springer-Verlag, (1992)

[LT] R. Longoni, T. Tradler, Homotopy inner products for cyclic operads, AT/0312231, (2003)

[MS1] J.E. McClure, J.H. Smith, A solution of Deligne's Hochschild cohomology conjecture, Contemp. Math.,Vol 293, 153-193, (2002) 
[MS2] J.E. McClure, J.H. Smith, Operads and cosimplicial objects: an introduction, Axiomatic, enriched and motivic homotopy theory, 133-171, NATO Sci. Ser. II Math. Phys. Chem., 131, Kluwer Acad. Publ., Dordrecht, 2004

$[\mathrm{M}] \quad$ L. Menichi, Batalin-Vilkovisky algebras and cyclic cohomology of Hopf algebras, $K$-Theory 32 (2004), no. 3, 231-251

[St] J. Stasheff, On the homotopy associativity of H-spaces I and II, Trans. AMS 108, p. 275-312, (1963)

[Su] D. Sullivan, Open and closed string field theory interpreted in classical algebraic topology, Topology, geometry and quantum field theory, 344-357, London Math. Soc. Lecture Note Ser., 308, Cambridge Univ. Press, Cambridge, (2004)

[Ta1] D. Tamarkin, Another proof of M. Kontsevich formality theorem, QA/9803025, (1998)

[Ta2] D. Tamarkin, Formality of chain operad of little discs, Lett. Math. Phys. 66 (2003), no. 1-2, 65-72

[Tr1] T. Tradler, Infinity-inner-products on A-infinity algebras, AT/0108027, (2001)

[Tr2] T. Tradler, The BV algebra on Hochschild cohomology induced by infinity inner products, QA/0210150, (2002)

[TZ1] T. Tradler, M. Zeinalian, Poincare duality at the chain level, and a $B V$ structure on the homology of the free loop space of a simply connected Poincare duality space, math.AT/0309455. (2003)

[TZ2] T. Tradler, M. Zeinalian, On the cyclic Deligne conjecture, J. Pure and Appl. Alg., 204 (2006) 280-299

[V] A.A. Voronov, Homotopy Gerstenhaber algebras, Confrence Mosh Flato 1999, Vol. II (Dijon), 307-331, Math. Phys. Stud., 22, Kluwer Acad. Publ., Dordrecht, (2000)

Thomas Tradler, Department of Mathematics, College of Technology of the City University of New York, 300 Jay Street, Brooklyn, NY 11201, USA

E-mail address: ttradler@citytech.cuny.edu

Mahmoud Zeinalian, Department of Mathematics, C.W. Post Campus of Long Island University, 720 Northern Boulevard, Brookville, NY 11548, USA

E-mail address: mzeinalian@liu.edu 